Supporting Information for

\title{
In situ generation of the surface oxygen vacancies in copper-ceria catalyst for the water-gas shift reaction
}

Wen-Zhu Yu, Mei-Yao Wu, Wei-Wei Wang*, Chun-Jiang Jia*

Key Laboratory for Colloid and Interface Chemistry, Key Laboratory of Special Aggregated Materials, School of Chemistry and Chemical Engineering, Shandong University, Jinan, 250100, China

*Corresponding author: jiacj@sdu.edu.cn,wangww@ @sdu.edu.cn. 


\section{Table of Contents}

1) Table S1. The surface area, copper content and $\mathrm{H}_{2}$ consumption of different samples.

2) Figure S1. The TEM images of (a) the $2 \mathrm{CuCe}-\mathrm{NR}$-dry sample, (b) the $2 \mathrm{CuCe}-\mathrm{NP}$-dry sample, (c) the fresh 2CuCe-NR-400 sample, (d) the fresh 2CuCe-NP-400 sample, (e) the fresh 2CuCe-NR-800 sample, (f) the fresh $2 \mathrm{CuCe}-\mathrm{NP}-800$ sample, $(\mathrm{g})$ the fresh $\mathrm{CeO}_{2}-\mathrm{NR}-800$ sample, and (h) the fresh $\mathrm{CeO}_{2}-\mathrm{NP}-800$ sample.

3) Figure S2. The TEM images of (a) the 10CuCe-NR-400 sample, (b) the 10CuCe-NR-800 sample (The orange circles were used to emphasize the $\mathrm{CuO}$ particles). (c) XRD diffraction pattern, (d) Raman spectrum, and (e, f) $\mathrm{H}_{2}$-TPR pattern of $5 \mathrm{CuCe}-\mathrm{NR}$ and $10 \mathrm{CuCe}-\mathrm{NR}$ with different calcination temperature.

4) Figure S3. Temperature-dependent $\mathrm{CO}$ conversion over (a) different $2 \mathrm{CuCe}-\mathrm{NR}$ catalysts, (b) different $2 \mathrm{CuCe}-\mathrm{NP}$ catalysts (Reaction conditions: $100 \mathrm{mg},[\mathrm{CO}]=2 \%,\left[\mathrm{H}_{2} \mathrm{O}\right]=10 \%$, the rest is argon, gas flow rate $=$ $\left.70 \mathrm{~mL} \cdot \mathrm{min}^{-1}\right)$.

5) Figure S4. TEM images for the fresh copper-ceria catalysts: (a) $2 \mathrm{CuCe}-\mathrm{NC}-400$ and (b) $2 \mathrm{CuCe}-\mathrm{NC}-800$. (c) Temperature-dependent $\mathrm{CO}$ conversion over 2CuCe-NC-400 and 2CuCe-NC-800 for the WGS reaction (Reaction conditions: $100 \mathrm{mg},[\mathrm{CO}]=2 \%,\left[\mathrm{H}_{2} \mathrm{O}\right]=10 \%$, the rest is argon, gas flow rate $=70 \mathrm{~mL} \cdot \mathrm{min}^{-1}$ ).

6) Figure S5. Kinetic orders of the (a) $\mathrm{CO}$ and (b) $\mathrm{H}_{2} \mathrm{O}$ for $2 \mathrm{CuCe}-\mathrm{NR}-400,2 \mathrm{CuCe}-\mathrm{NR}-800$ and $2 \mathrm{CuCe}-\mathrm{NP}-800$ at variable temperatures.

7) Figure S6. The TPSR profiles over 2CuCe-NR-y and 2CuCe-NP-800: (a, d, g) CO-TPSR in intensity, (b, e, h) CO-TPSR in concentration and (c, f, i) dissociation of $\mathrm{H}_{2} \mathrm{O}$; (a-c) for 2CuCe-NR-400, (d-f) for 2CuCe-NR-800, and (g-i) for 2CuCe-NP-800.

8) Figure S7. In situ DRIFTS spectra of time-sequencing (a) -OD region and (b) CO region collected under CO injection for the $2 \mathrm{CuCe}-\mathrm{NR}-800$ catalyst. 
Table S1. The surface area, copper content and $\mathrm{H}_{2}$ consumption of different samples.

\begin{tabular}{cccc}
\hline Samples & $A_{\text {BET }}\left(\mathrm{m}^{2} \cdot \mathrm{g}^{-1}\right)$ & Copper content $(\%)$ & $\mathrm{H}_{2}$ consumption $\left(\mu \mathrm{mol} \cdot \mathrm{g}^{-1}\right)$ \\
\hline $\mathrm{CeO}_{2}$-NR-400 & 92.3 & -- & -- \\
$\mathrm{CeO}_{2}$-NP-400 & 106.5 & -- & - \\
$\mathrm{CeO}_{2}$-NR-800 & 4.9 & -- & -- \\
$\mathrm{CeO}_{2}$-NP-800 & 2.9 & -- & -- \\
$2 \mathrm{CuCe}-\mathrm{NR}-400$ & 94.3 & 2.1 & 1440 \\
$2 \mathrm{CuCe}-\mathrm{NR}-800$ & 28.1 & 2.2 & 796 \\
$2 \mathrm{CuCe}-\mathrm{NP}-400$ & 104.3 & 2.3 & 1876 \\
$2 \mathrm{CuCe}-\mathrm{NP}-800$ & 5.2 & 2.2 & 845 \\
\hline
\end{tabular}

Note: The theoretical $\mathrm{H}_{2}$ consumption of $\mathrm{CuO}$ is about $312 \mu \mathrm{mol} \cdot \mathrm{g}^{-1}$. 

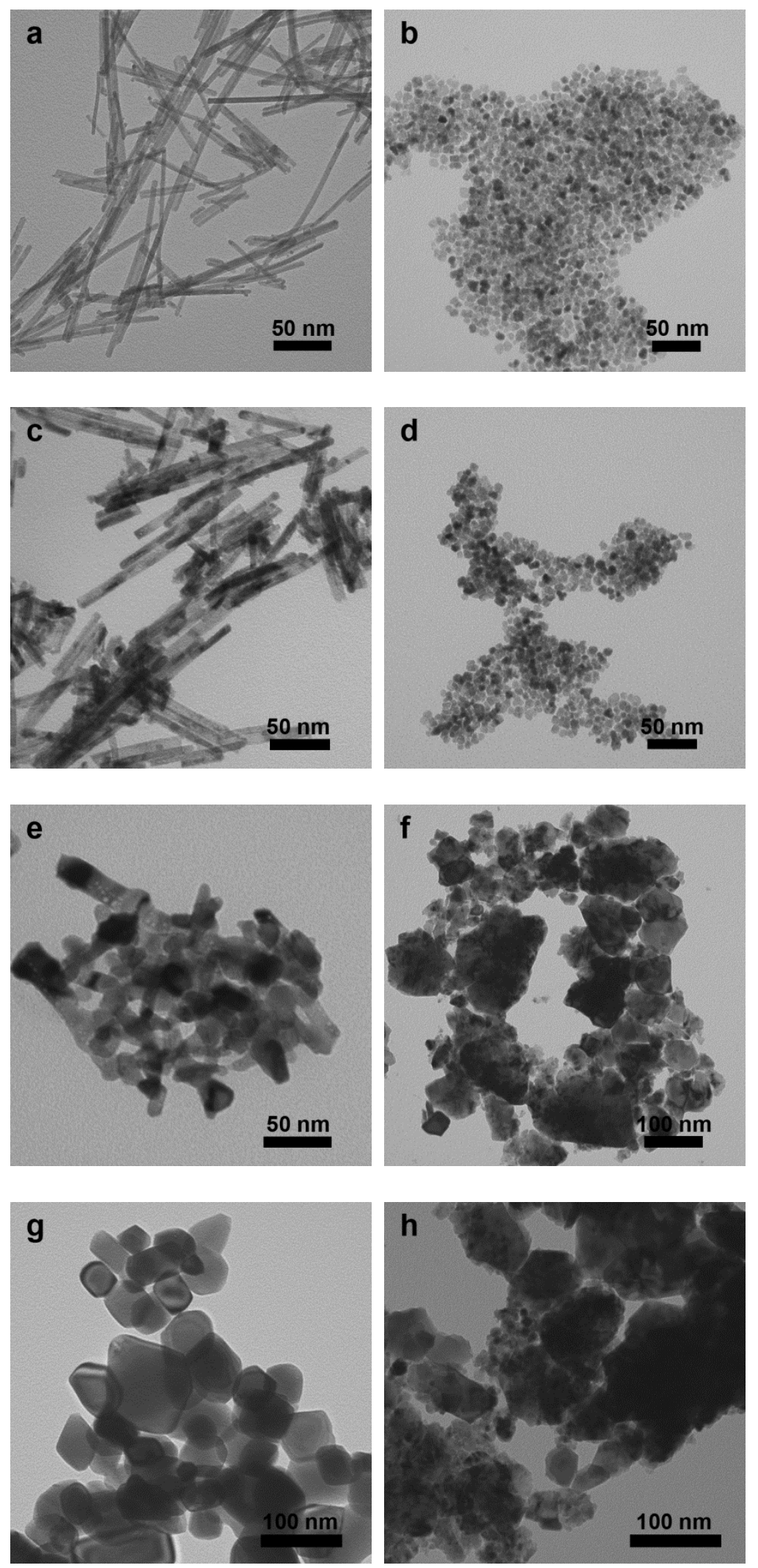

Figure S1. The TEM images of (a) the 2CuCe-NR-dry sample, (b) the 2CuCe-NP-dry sample, (c) the fresh 2CuCe-NR-400 sample, (d) the fresh 2CuCe-NP-400 sample, (e) the fresh 2CuCe-NR-800 sample, (f) the fresh 2CuCe-NP-800 sample, (g) the fresh $\mathrm{CeO}_{2}-\mathrm{NR}-800$ sample, and (h) the fresh $\mathrm{CeO}_{2}-\mathrm{NP}-800$ sample. 

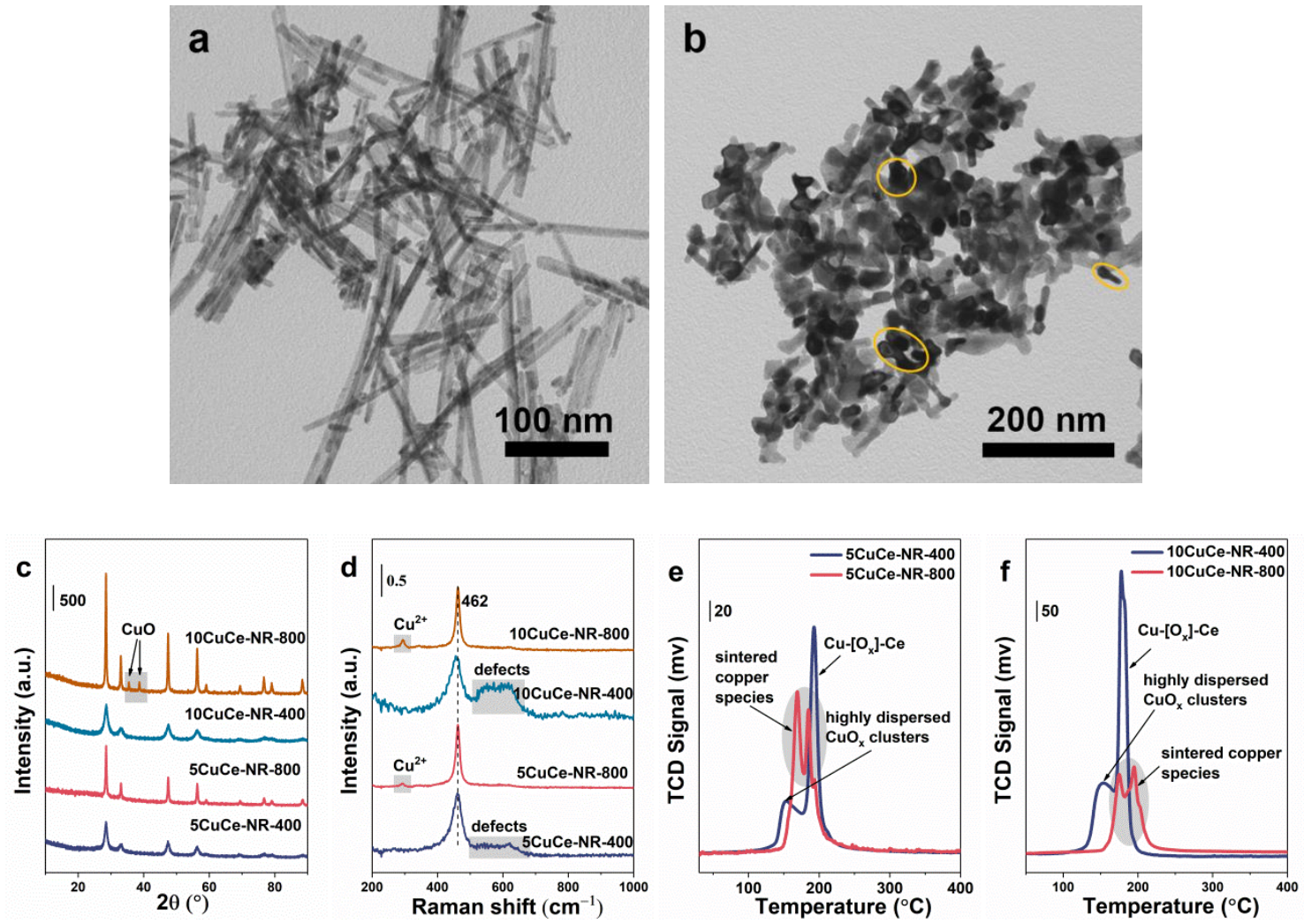

Figure S2. The TEM images of (a) the $10 \mathrm{CuCe}-\mathrm{NR}-400$ sample, (b) the $10 \mathrm{CuCe}-\mathrm{NR}-800$ sample (The orange circles were used to emphasize the $\mathrm{CuO}$ particles). (c) XRD diffraction pattern, (d) Raman spectrum, and (e, f) $\mathrm{H}_{2}$-TPR pattern of 5CuCe-NR and 10CuCe-NR with different calcination temperature. 

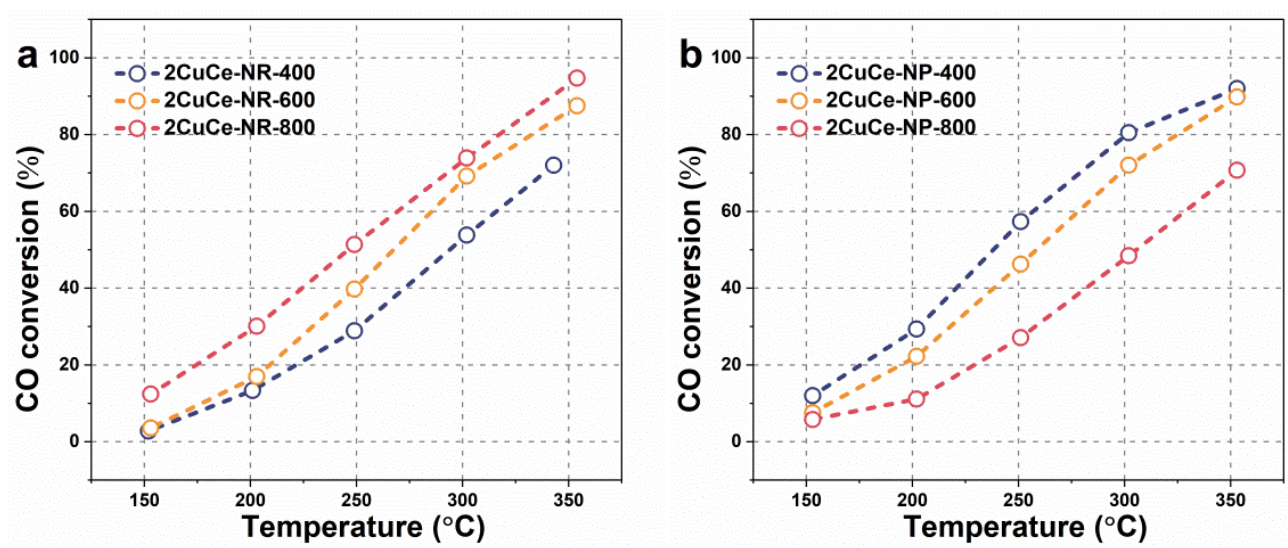

Figure S3. Temperature-dependent CO conversion over (a) different 2CuCe-NR catalysts, (b) different 2CuCe-NP catalysts (Reaction conditions: $100 \mathrm{mg},[\mathrm{CO}]=2 \%,\left[\mathrm{H}_{2} \mathrm{O}\right]=10 \%$, the rest is argon, gas flow rate $=70 \mathrm{~mL} \cdot \mathrm{min}^{-1}$ ). 

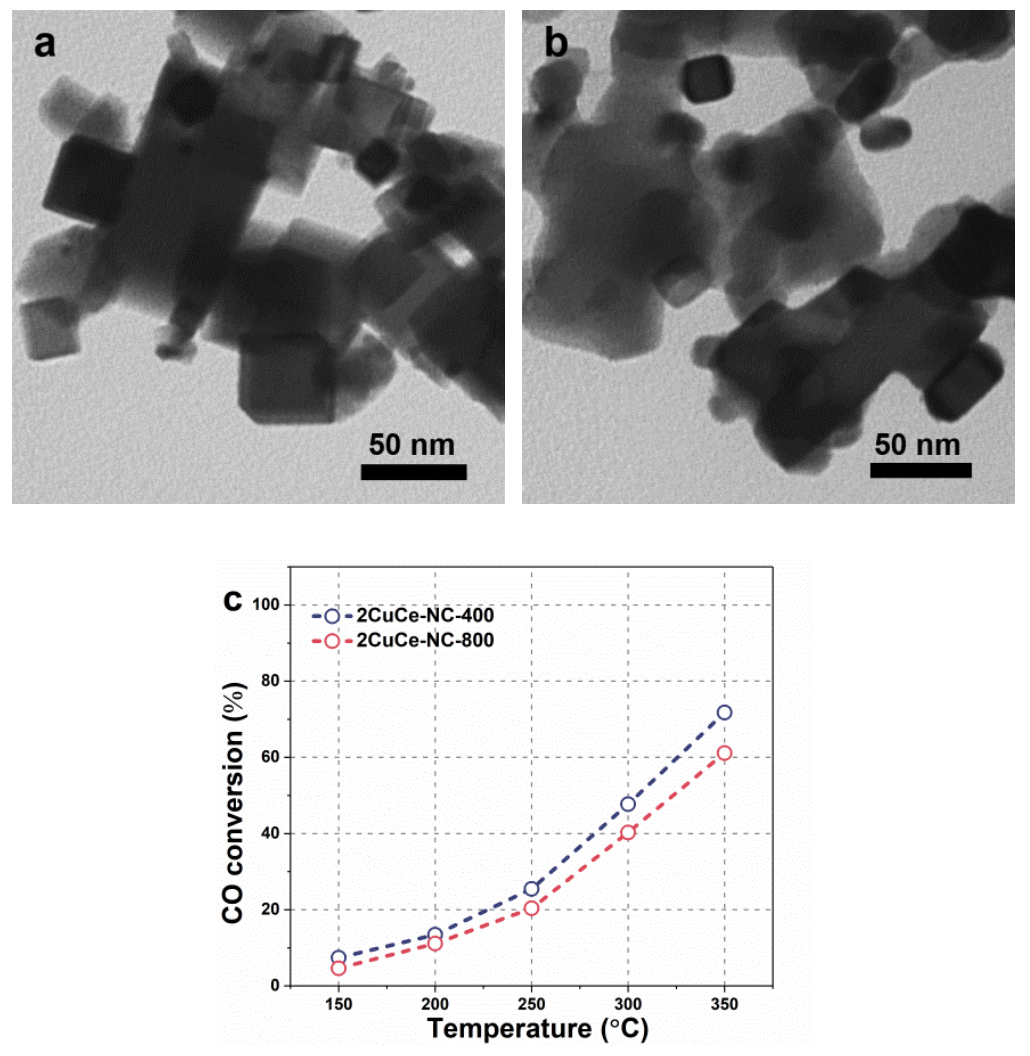

Figure S4. TEM images for the fresh copper-ceria catalysts: (a) 2CuCe-NC-400 and (b) 2CuCe-NC-800. (c) Temperature-dependent $\mathrm{CO}$ conversion over $2 \mathrm{CuCe}-\mathrm{NC}-400$ and $2 \mathrm{CuCe}-\mathrm{NC}-800$ for the WGS reaction (Reaction conditions: $100 \mathrm{mg},[\mathrm{CO}]=2 \%,\left[\mathrm{H}_{2} \mathrm{O}\right]=10 \%$, the rest is argon, gas flow rate $=70 \mathrm{~mL} \cdot \mathrm{min}^{-1}$ ). 

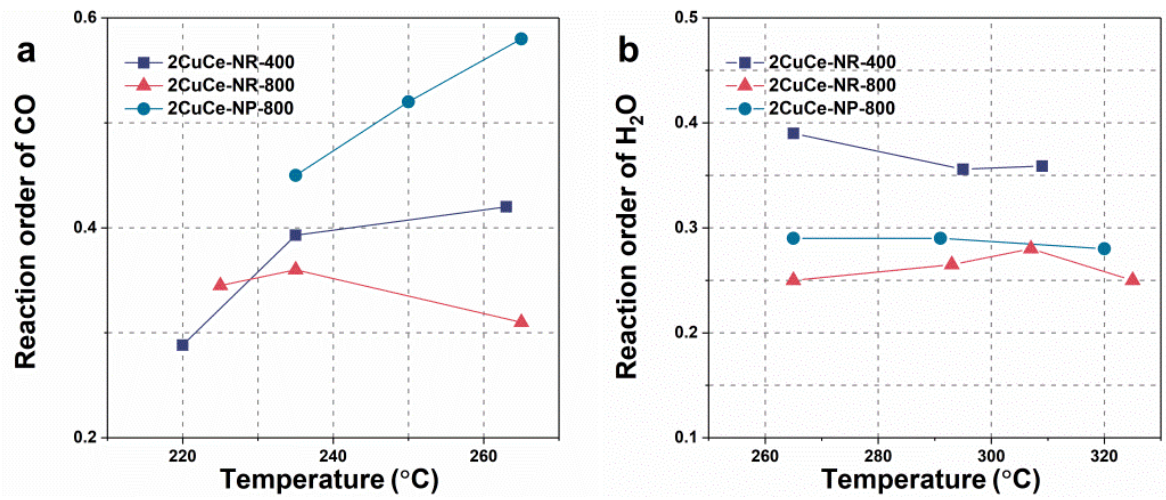

Figure S5. Kinetic orders of the (a) $\mathrm{CO}$ and (b) $\mathrm{H}_{2} \mathrm{O}$ for $2 \mathrm{CuCe}-\mathrm{NR}-400,2 \mathrm{CuCe}-\mathrm{NR}-800$ and $2 \mathrm{CuCe}-\mathrm{NP}-800$. 

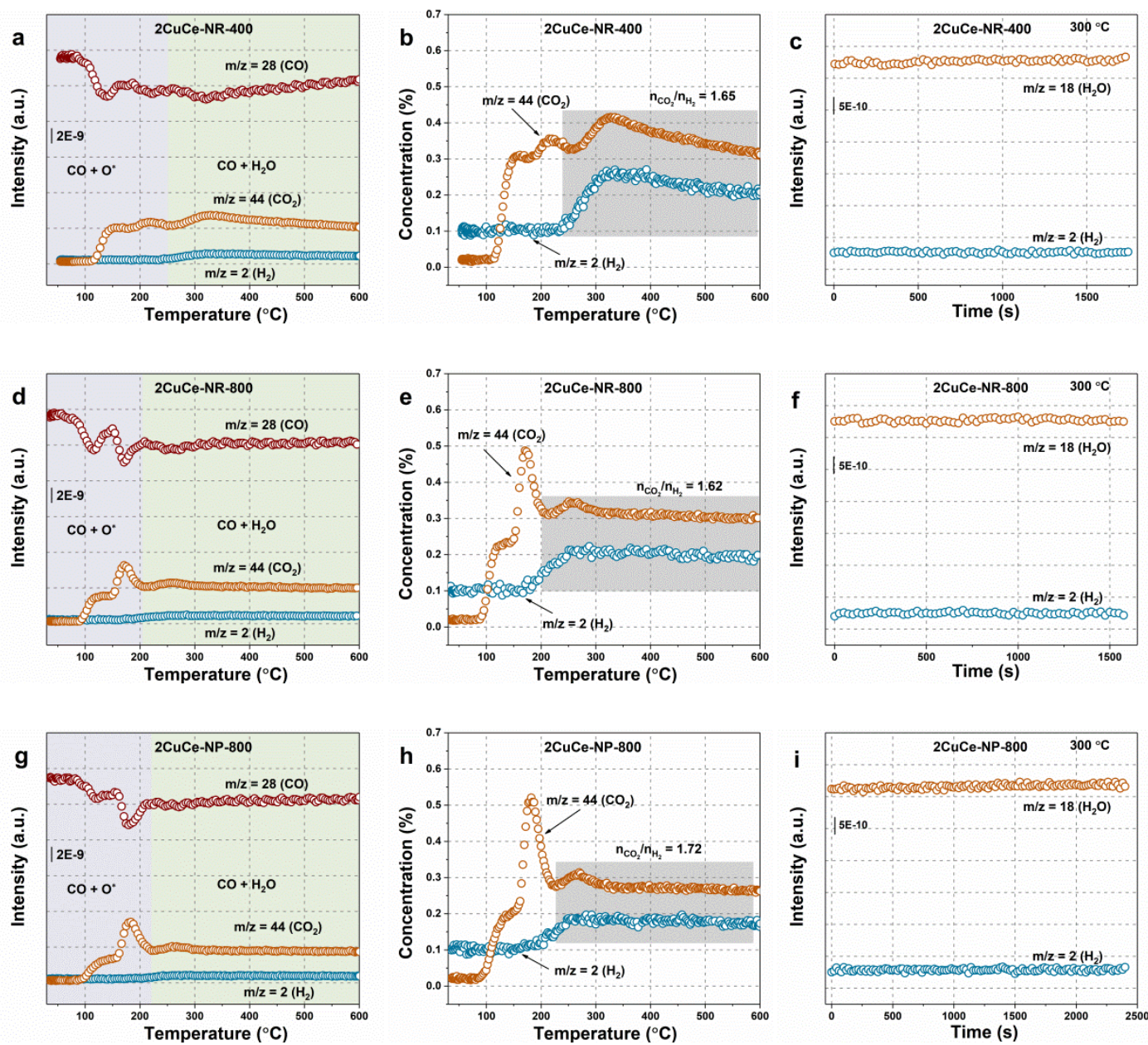

Figure S6. The TPSR profiles over 2CuCe-NR-y and 2CuCe-NP-800: (a, d, g) CO-TPSR in intensity, (b, e, h) CO-TPSR in concentration and (c, f, i) dissociation of $\mathrm{H}_{2} \mathrm{O}$; (a-c) for 2CuCe-NR-400, (d-f) for 2CuCe-NR-800, and $(\mathrm{g}-\mathrm{i})$ for $2 \mathrm{CuCe}-\mathrm{NP}-800$. 

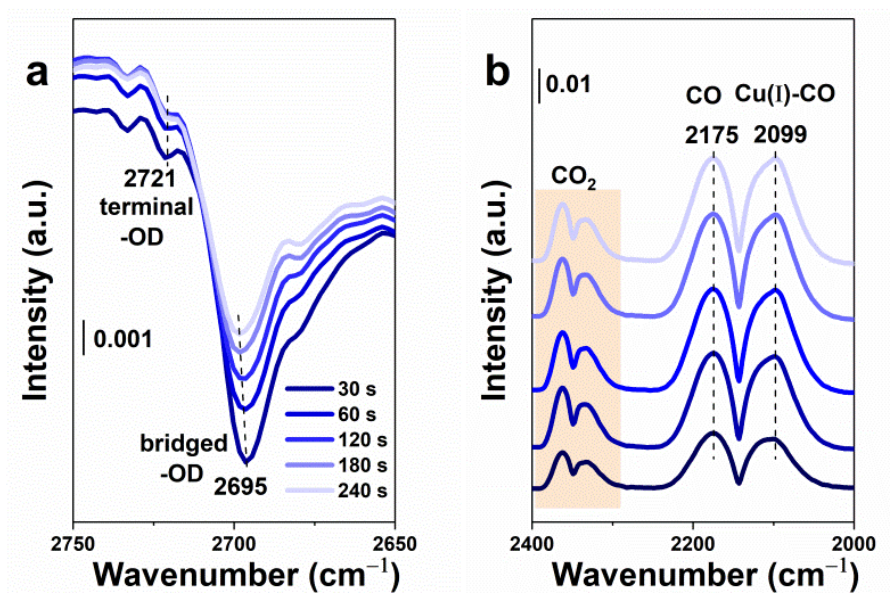

Figure S7. In situ DRIFTS spectra of time-sequencing (a) -OD region and (b) CO region collected under CO injection for the $2 \mathrm{CuCe}-\mathrm{NR}-800$ catalyst. 